Pacific Journal of Mathematic 


\title{
A THEOREM ON QUASI-PURE-PROJECTIVE TORSION FREE ABELIAN GROUPS OF FINITE RANK
}

\author{
C. Vinsonhaler AND W. WickLESS
}

It is shown that a quasi-pure-projective torsion free abelian group of finite rank is either strongly indecomposable or a direct sum of isomorphic rank one groups.

The purpose of this note is to record the author's theorem that quasi-pure-projective (qpp) torsion free abelian groups of finite rank are either strongly indecomposable or homogeneous completely decomposable.

This result has been in the folklore for several years. It is cited in [1], Theorem E (a classification theorem for finite rank qpp groups) and in [3], (a characterization of locally free quasi-pure-injective groups).

Throughout, "group" will mean torsion free abelian group of finite rank. Otherwise the terminology and notations are from [2].

Definition 1. A group $A$ is a quasi-pure-projective if for any pure subgroup $K$ of $A$ and homomorphism $\theta: A \rightarrow A / K$, there is an endomorphism $\bar{\theta}$ of $A$ such that $\pi \bar{\theta}=\theta$, where $\Pi: A \rightarrow A / K$ is the natural factor map.

For the proof of our result it is convenient to consider what seems to be a generalization of the qpp property. (Actually, in [3] it is shown that the property defined below is equivalent to qpp in the finite rank case).

Definition 2. A group $A$ is almost quasi-pure-projective (aqpp) if there exists a fixed integer $n$ such that for any pure subgroup $K$ of $A$ and homomorphism $\theta: A \rightarrow A / K$, there is an endomorphism $\bar{\theta}$ of $A$ such that $\Pi \bar{\theta}=n \theta$, where $\Pi: A \rightarrow A / K$ is the natural factor map.

The integer $n$ will be called the associated integer for the aqpp group $A$.

A sequence of lemmas leads to the result. The proof of the first one is routine.

Lemma 1. A direct summand of an aqpp group is aqpp.

The proof of the next lemma is given in [3], §5. The proof can also be obtained by slight modification of Lemma 4.1 of [1]. 
LemMa 2. Let $A$ be a strongly indecomposable aqpp group and $B$ be a proper pure subgroup of $A$. Then $A$ and $A / B$ are divisible by the same primes.

Let $\left\langle g_{1}, g_{2}, \cdots, g_{n}\right\rangle_{*}$ denote the pure subgroup generated by the elements $g_{1}, g_{2}, \cdots, g_{n}$. (The group in which the pure subgroup is to be taken will be obvious in each instance where the notation is used.)

The next lemma is the key to the theorem.

LEMMA 3. Let $A$ and $B$ be strongly indecomposable reduced groups such that $A \oplus B$ is aqpp. Then given $0 \neq x \in A$ and $0 \neq y \in$ $B$, there is a homomorphism $f: B \rightarrow\langle x\rangle_{*}$ such that $f(y) \neq 0$.

Proof. Let $p$ be a prime such that $p B \neq B$, and write $n=p^{k} l$, with $(p, l)=1$, where $n$ is the associated integer for $A \oplus B$. Now let $\left\{y=y_{1}, y_{2}, \cdots, y_{s}\right\}$ be a maximal rationally independent set in $B$ and let $B_{1}=\left\{q_{1} \mid q_{1} y_{1}+\cdots+q_{s} y_{s} \in B, q_{j} \in Q, 1 \leqq j \leqq s\right\}$. Since $B_{1} \cong$ $B /\left\langle y_{2}, \cdots, y_{s}\right\rangle_{*}$, Lemmas 1 and 2 imply that $p B_{1} \neq B_{1}$. Let $t$ be the largest integer such that $1 / p^{t} \in B_{1}$. Form $H=\left\langle p^{k+2 t+1} x-y_{1}\right.$, $\left.y_{2}, y_{3}, \cdots, y_{s}\right\rangle_{*}$ and consider the map $\theta: A \oplus B \rightarrow(A \oplus B) / H$ given by $\theta(a \oplus b)=\beta_{1} / p^{k+t+1}\left(y_{1}+H\right)$ where $a \in A$ and $b=\sum_{j=1}^{s} \beta_{j} y_{j} \in B$. Note that $\theta$ is defined since $y_{1}+H=p^{k+2 t+1}(x+H)$. Since $A \oplus B$ is aqpp, there is a lifting $\bar{\theta}: A \oplus B \rightarrow A \oplus B$ such that $\Pi \bar{\theta}=n \theta$, where $\Pi$ : $A \oplus B \rightarrow(A \oplus B) / H$ is the natural projection. Then $p^{t+1} \bar{\theta}\left(y_{1}\right)$ has the form $p^{t+1} \bar{\theta}\left(y_{1}\right)=n p^{t+1} /\left(p^{k+t+1}\right) y_{1}+h=l y_{1}+h$ where $h \in H$.

Let $\Pi_{A}$ be projection of $A \oplus B$ onto $A$. If $\Pi_{A} \bar{\theta}\left(y_{1}\right)=0$, then in the above equation, $h \in B$. But then $1 / p^{t+1}\left(l y_{1}+h\right) \in B$, contradicting the choice of $t$. Thus $0 \neq \Pi_{A} \bar{\theta}\left(y_{1}\right) \in \Pi_{A} \bar{\theta}(B) \cong\langle x\rangle_{*}$.

The final lemma is the motivation for considering aqpp groups. The proof is routine.

Lemma 4. If $A$ is quasi-isomorphic to $B(A \sim B)$ and $A$ is aqpp, then $B$ is aqpp.

Our theorem now follows easily, using Lemmas $1-4$.

THEOREM. Let $A$ be an aqpp group. Then $A$ is either strongly indecomposable or is a direct sum of isomorphic groups of rank one.

Proof. If $A$ is not strongly indecomposable, write $A \sim A_{1} \oplus \cdots \oplus$ $A_{r}, r \geqq 2$, where each $A_{i}$ is nonzero and strongly indecomposable 
([2]), §92). Given $0 \neq y \in A_{1}, 0 \neq x \in A_{2}$, apply Lemmas 3 and 4 to obtain $f: A_{1} \rightarrow\langle x\rangle_{*}$ with $f(y) \neq 0$ and (by symmetry) $g: A_{2} \rightarrow\langle y\rangle_{*}$ with $g(x) \neq 0$. We conclude that type $(x)=$ type $(y)$ and $\langle x\rangle_{*} \cong\langle y\rangle_{*}$ ([2], p. 109 (D) and Th. 85.1).

But if $f: A_{1} \rightarrow\langle x\rangle_{*}$ with $f(y) \neq 0$ and type $(x) \equiv$ type $(y)$, then it immediately follows that $\langle y\rangle_{*}$ is a quasi-summand of $A_{1}$. To see this let $K=\operatorname{Ker} f$ and note that $\langle y\rangle_{*} \cap K=(0)$. Since $A_{1} / K$ is isomorphic to a subgroup of $\langle x\rangle_{*}$ we have: type $\langle y\rangle_{*}=$ type $\left(\langle y\rangle_{*} \oplus\right.$ $K) / K \leqq$ type $A_{1} / K \leqq$ type $\langle x\rangle_{*}=$ type $\langle y\rangle_{*}$. Thus, there exists an integer $m$ such that $m\left(A_{1} / K\right) \subseteq\left(\langle y\rangle_{*} \oplus K\right) / K$. This gives $A_{1} \sim\langle y\rangle_{*} \oplus$ $K$. Hence $A_{1}=\langle y\rangle_{*}$. Similarly, all of the $A_{i}$ must be of rank one, and having the same type, are isomorphic. Thus $A$ is quasi-isomorphic to a direct sum of isomorphic groups of rank one. This implies that $A$ is actually isomorphic to this sum by [2], Prop. 98.1.

\section{REFERENCES}

1. D. Arnold, B. O'Brien and J. Reid, Quasi-pure injective and projective torsion free Abelian groups of finite rank, London Math. Soc. Proc., 38 (1979), 532-544.

2. L. Fuchs, Infinite Abelian Groups, Academic Press, New York.

3. C. Vinsonhaler, Almost quasi-pure injective Abelian groups, Rocky Mt. J. Math., 9 (1979), 569-576.

Received February 6, 1979 and in revised form April 2, 1980.

The University of Connecticut

Storrs, CT 06268 



\section{PACIFIC JOURNAL OF MATHEMATICS}

\section{EDITORS}

DoNALD BABBITT (Managing Editor)

University of Galifornia

Los Angeles, California 90024

HUgo RossI

University of Utah

Salt Lake City, UT 84112

C. C. MOORE AND ANDREW OGG

University of California

Berkeley, CA 94720
J. DugunduI

Department of Mathematics

University of Southern California

Los Angeles, California 90007

R. Finn and J. Milgram

Stanford University

Stanford, California 94305

\section{ASSOCIATE EDITORS}
E. F. BeCKenbaCh
B. H. NeumanN
F. WOLF
K. YoSHIDA

\section{SUPPORTING INSTITUTIONS}

UNIVERSITY OF BRITISH COLUMBIA

CALIFORNIA INSTITUTE OF TECHNOLOGY

UNIVERSITY OF CALIFORNIA

MONTANA STATE UNIVERSITY

UNIVERSITY OF NEVADA, RENO

NEW MEXICO STATE UNIVERSITY

OREGON STATE UNIVERSITY

UNIVERSITY OF OREGON
UNIVERSITY OF SOUTHERN CALIFONIA STANFORD UNIVERSITY UNIVERSITY OF HAWAII UNIVERSITY OF TOKYO UNIVERSITY OF UTAH WASHINGTON STATE UNIVERSITY UNIVERSITY OF WASHINGTON 


\section{Pacific Journal of Mathematics}

\section{Vol. 91, No. $1 \quad$ November, 1980}

Harvey Leslie Abbott, Extremal problems on nonaveraging and nondividing

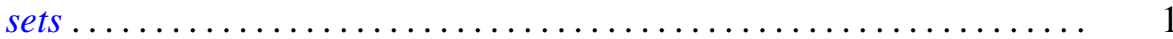

Marine Bruce Abrahamse and Stephen D. Fisher, Mapping intervals to

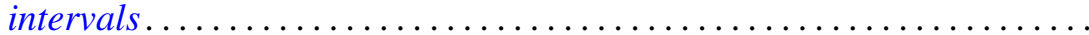

William Wells Adams, The best two-dimensional Diophantine

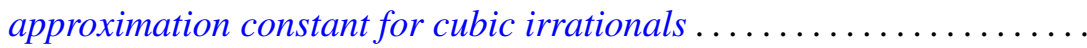

Marilyn Breen, A quantitative version of Krasnosel'skiu 's theorem in

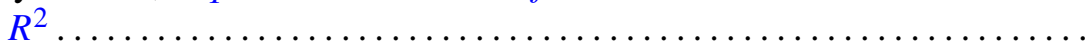

Stephen LaVern Campbell, Linear operators for which $T^{*} T$ and $T T^{*}$

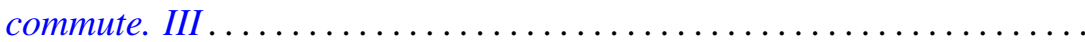

Zvonko Cerin, On cellular decompositions of Hilbert cube manifolds ......

J. R. Choike, Ignacy I. Kotlarski and V. M. Smith, On a characterization

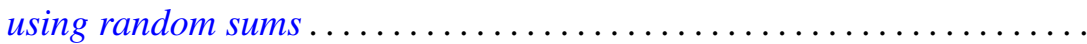

Karl-Theodor Eisele, Direct factorizations of measures .............. 79

Douglas Harris, Every space is a path component space ............. 95

John P. Holmes and Arthur Argyle Sagle, Analytic H-spaces, Campbell-Hausdorff formula, and alternative algebras.............

Richard Howard Hudson and Kenneth S. Williams, Some new residuacity criteria ..........................................

V. Karunakaran and Michael Robert Ziegler, The radius of starlikeness for a class of regular functions defined by an integral ....

Ka-Sing Lau, On the Banach spaces of functions with bounded upper

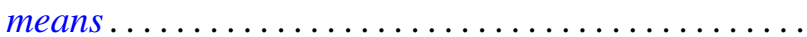

Daniel Paul Maki, On determining regular behavior from the recurrence formula for orthogonal polynomials................

Stephen Joseph McAdam, Asymptotic prime divisors and going down...

Douglas Edward Miller, Borel selectors for separated quotients ..

Kent Morrison, The scheme of finite-dimensional representations of an algebra

Donald P. Story, A characterization of the local Radon-Nikodým property by tensor products

Arne Stray, Two applications of the Schur-Nevanlinna algorithm ...

N. B. Tinberg, The Levi decomposition of a split $(B, N)$-pair ...

Charles Irvin Vinsonhaler and William Jennings Wickless, A theorem on quasi-pure-projective torsion free abelian groups of finite rank... 